\title{
Chikungunya virus was isolated in Thailand, 2010
}

\author{
Mikiko Sasayama $\cdot$ Surachet Benjathummarak $\cdot$ Norihito Kawashita $\cdot$ \\ Prasert Rukmanee $\cdot$ Suntaree Sangmukdanun · Promsin Masrinoul • \\ Pannamthip Pitaksajjakul • Orapim Puiprom • Pitak Wuthisen • \\ Takeshi Kurosu • Panjaporn Chaichana - Pannamas Maneekan • \\ Kazuyoshi Ikuta $\cdot$ Pongrama Ramasoota $\cdot$ Tamaki Okabayashi $\cdot$ \\ Pratap Singhasivanon $\cdot$ Natthanej Luplertlop
}

Received: 22 March 2014/ Accepted: 21 July 2014/Published online: 12 August 2014

(C) The Author(s) 2014. This article is published with open access at Springerlink.com

\begin{abstract}
Chikungunya fever (CHIKF) is an acute febrile illness caused by a mosquito-borne alphavirus, chikungunya virus (CHIKV). This disease re-emerged in Kenya in 2004, and spread to the countries in and around the Indian Ocean. The re-emerging epidemics rapidly spread to regions like India and Southeast Asia, and it was subsequently identified in Europe in 2007, probably as a result of importation of chikungunya cases. On the one hand, chikungunya is one of the neglected diseases and has only attracted strong attention during large outbreaks. In 2008-2009, there was a major outbreak of chikungunya fever in Thailand, resulting in the highest number of infections in any country in the region. However, no update
\end{abstract}

Mikiko Sasayama and Surachet Benjathummarak have contributed equally to this work.

Electronic supplementary material The online version of this article (doi:10.1007/s11262-014-1105-5) contains supplementary material, which is available to authorized users.

M. Sasayama · P. Masrinoul · O. Puiprom - P. Chaichana . T. Okabayashi

Mahidol-Osaka Center for Infectious Diseases, Ratchathewi,

Bangkok 10400, Thailand

M. Sasayama $\cdot$ T. Okabayashi

Research Institute for Microbial Diseases, Osaka University,

Suita, Osaka 565-0871, Japan

S. Benjathummarak · P. Pitaksajjakul · P. Ramasoota $(\bowtie)$ Center of Excellence for Antibody Research, Faculty of Tropical

Medicine, Mahidol University, Ratchathewi, Bangkok 10400,

Thailand

e-mail: pongrama.ram@mahidol.ac.th

N. Kawashita

Department of Environmental Pharmacometrics, Graduate

School of Pharmaceutical Sciences, Osaka University, Suita,

Osaka 565-0871, Japan of CHIKV circulating in Thailand has been published since 2009. In this study, we examined the viral growth kinetics and sequences of the structural genes derived from CHIKV clinical isolates obtained from the serum specimens of CHIKF-suspected patients in Central Thailand in 2010. We identified the CHIKV harboring two mutations E1-A226V and E2-I211T, indicating that the East, Central, and South African lineage of CHIKV was continuously circulating as an indigenous population in Thailand.

Keywords Chikungunya virus · Thailand · Virus replication - Genetic variation

\section{Introduction}

Chikungunya virus (CHIKV; family Togaviridae; genus Alphavirus) is a mosquito-borne, single-stranded, positive-

P. Rukmanee - S. Sangmukdanun - P. Wuthisen - P. Maneekan ·

P. Singhasivanon $\cdot$ N. Luplertlop $(\bowtie)$

Department of Tropical Hygiene, Faculty of Tropical Medicine,

Mahidol University, Ratchathewi, Bangkok 10400, Thailand

e-mail: natthanej.lup@mahidol.ac.th

T. Kurosu $\cdot$ K. Ikuta

Department of Virology, Research Institute for Microbial

Diseases, Osaka University, Suita, Osaka 565-0871, Japan

Present Address:

N. Luplertlop

Department of Microbiology and Immunology, Faculty of

Tropical Medicine, Mahidol University, Ratchathewi,

Bangkok 10400, Thailand 
sense RNA virus that is the causative agent of chikungunya fever (CHIKF), which is a major public health problem in Africa, South America, and Southern and Southeastern Asia $[1,2]$. CHIKV was first identified in Tanzania in the 1950s, and recent huge outbreak occurred in Kenya in 2004. This re-emerged epidemic subsequently spread to several countries in the Indian Ocean and India, with documented outbreak in Italy in 2007 [3]. The outbreak in New Caledonia in 2011 and the ongoing outbreak in Caribbean in 2013-2014 raise public health concerns again due to increasing number of CHIKV infections [4, 5]. There are three distinct lineages of CHIKV: the West African genotype; the East, Central, and South African (ECSA) genotype; and the Asian genotype.

In Thailand, outbreaks of chikungunya were first documented in the early 1960s, and the most recent outbreak was reported in 2008-2009 [6]. Classical CHIKF is characterized by high fever, nausea, rash, and severe arthralgia; however, the clinical signs and symptoms of CHIKF are indistinguishable from those of dengue, and both illnesses are transmitted by Aedes mosquitoes, such as Ae. aegypti and Ae. albopictus [7, 8].

Studies of the clinical and molecular characteristics of CHIKV during the recent outbreak of chikungunya in Thailand indicated that the virus belonged to the ECSA genotype, which was reported in the Indian Ocean islands after 2005 [9]. These isolates harbored the E1-A226V mutation, which increases virus transmissibility via Ae. albopictus [10,11]. By December 2009, CHIKV had spread to the Central, Northeastern, and Northern provinces of Thailand, infecting more than 46,000 individuals [1]. No study of CHIKV clinical isolates in Thailand has been published since 2009; therefore, we need to remain vigilant for the endemic situation of chikungunya in Thailand. Here, we examined the viral growth kinetics and sequences of the structural genes derived from CHIKV clinical isolates obtained from the serum specimens of CHIKF-suspected patients in Ratchaburi Province (100 km west of Bangkok) in 2010.

\section{Results and discussion}

The Royal Thai Government and the Faculty of Tropical Medicine at Mahidol University (MU-TropMed) are currently monitoring emerging diseases (e.g., dengue and malaria) with a view to future control and prevention in Ratchaburi Province. The aim of the present study was to examine the endemic situation regarding CHIKV circulation in Thailand, and in Ratchaburi Province in particular. From August to September, 2010, the MU-TropMed and Health Promoting Hospital (HPH) in Ratchaburi Province obtained 50 serum samples from individuals suspected of harboring CHIKF. Permission regarding the use of anonymous specimens was granted by the Director of HPH, and this study was approved by the Ethics Committee at MU-TropMed (MUTM 2012-047-01). Here, we report the isolation and molecular characterization of seven clinical isolates of CHIKV, which were derived from Thai individuals showing characteristic symptoms.

All serum specimens were tested for CHIKV infection using the SD BIOLINE Chikungunya IgM rapid test kit (Standard Diagnostics, Kyonggi-do, Korea). Briefly, $50 \mu \mathrm{l}$ of serum sample was dropped into the well of the test device, and assay diluent was subsequently added. Test results were interpreted at $10 \mathrm{~min}$. We also performed real-time reverse transcription-PCR (RT-PCR) assay, targeting the $E 1$ gene, using the iScript One-Step RT-PCR Kit for Probes (Bio-Rad, CA, USA). Viral RNA was extracted from $140 \mu \mathrm{l}$ of serum sample using the QIAamp Viral RNA Mini Kit (QIAGEN, Hilden, Germany) according to manufacturer's instruction. The following primers were used for the real-time RT-PCR: CHIK-forward (AAGCTYCGCGTCCTTTACCAAG), CHIK-reverse (CCAAATTGTCCYGGTCTTCCT), and CHIK-probe (FAM-CCAATGTCYTCMGCCTGGACACCTTT-

TAMRA) as described previously [12].

Of the 50 serum samples tested, $6(12 \%)$ were identified as CHIKV positive by real-time RT-PCR, and one ( $2 \%)$ was identified as chikungunya positive by both real-time RT-PCR and Chikungunya IgM antibody test kit. All seven CHIKV-positive specimens were obtained from individuals in the acute phase of the illness ( $\leq 6$ days post-onset). We isolated CHIKV using an Ae. albopictus-derived cell line, $\mathrm{C} 6 / 36$, and the supernatants of individual clinical isolates were used for further study.

We first examined the viral growth kinetics of two clinical isolates (CP9 and CP11) and three control strains (Ross strain_1953, \#32808_2008, and \#16856_2009) selected randomly. Vero and C6/36 cells were inoculated with these viruses at a multiplicity of infection of one. The time of inoculation was set as "hour 0". Culture supernatants were collected at 4, 8, 12, 24, and $28 \mathrm{~h}$ post-inoculation, and were titrated with plaque assay. The viral titers of the clinical isolates were significantly higher than those of the controls during the first $8 \mathrm{~h}$ post-inoculation in Vero cells $(P<0.0005)$ and the first $12 \mathrm{~h}$ post-inoculation in $C 6 / 36$ cells $(P<0.005)$ (Supplemental Fig. 1). In Vero cells, the peak viral titers for $\mathrm{CP} 9$ and $\mathrm{CP} 11$ at $12 \mathrm{~h}$ post-inoculation were $3.5 \times 10^{6}$ and $3.6 \times 10^{6} \mathrm{PFU} / \mathrm{mL}$, respectively. In C6/36 cells, the peak viral titers for $\mathrm{CP} 9$ and $\mathrm{CP} 11$ were $5.9 \times 10^{8}$ and $4.8 \times 10^{8}$ $\mathrm{PFU} / \mathrm{mL}$ at $24 \mathrm{~h}$ post-inoculation, respectively. We also examined the cytopathic effects (CPEs) of CP9, CP11, and the control viruses against $\mathrm{C} 6 / 36$ cells. Both isolates showed milder CPEs in C6/36 cells than the controls.

Next, to examine sequence variations within the viral genome, the seven clinical isolates were subjected to 
Fig. 1 a Phylogenetic tree showing the relationships between the structural genes of different chikungunya virus (CHIKV) strains. The information was obtained from 56 clones derived from 7 infected patients in Ratchaburi Province of Thailand in 2010. The numbers below or above the branch nodes represent the neighbor-joining bootstrap values. Analysis was based on the nucleotide sequences of the structural genes using the MEGA 5.05 program and the maximum likelihood method based on 1,000 bootstrap replications. The 2010 isolates are denoted by black circles and bold type. Scale bars indicate 0.02 nucleotide substitutions per site. GenBank Accession numbers for all isolates were recorded. GenBank Accession nos: CP1 (AB857730

AB857737); CP7 (AB857738AB857745); CP9 (AB857746AB857753); CP10 (AB857754 AB857761); CP11 (AB857762AB857769); CP13 (AB857770$\mathrm{AB} 857777)$; and CP16 (AB857778-AB857785),

b Three-dimensional structures of chikungunya E1, E2, and E3 monomers by ribbon model were obtained from the Protein Data Bank (ID code 3N41) [16]. The domains are color coded as follows: Domain I of E1 (dark red), domain II of E1 (dark yellow), domain III of E1 (dark blue), the FL region of $\mathrm{E} 1$ (orange), domain $\mathrm{A}$ of $\mathrm{E} 2$ (blue-green), domain $\mathrm{B}$ of $\mathrm{E} 2$ (dark green), domain $\mathrm{C}$ of $\mathrm{E} 2$ (pink), the arch region of $\mathrm{E} 2$ (purple), and E3 (gray).

Mutated residues are depicted as a space-filling model: $\mathrm{H} 456$ (red), E572 (magenta), P1113 (light green), N1158 (light blue), and sugar-binding residues (gray). Yellow in space-filling model shows the immunodominant site. This figure was prepared by Molecular Operating Environment (Chemical Computing Group Inc) (Color figure online) (a)

JX088705.1/C hina/2010

- HQ846357.1/C hina/2010

HQ846356.1/C hina/2010

63 FJ807895.1/Malaysia/2009

JQ065886.1/C hina/2010

FJ807893.1/Malaysia/2008

JQ861258.1/C ambodia/2011

9s JQ861259.1/C ambodia/2011

os JQ861260.1/C ambodia/2011 FJ807892.1/Malaysia/2008

FJ445443.2/Singapore/2008

FJ445484.2/Singapore/2008

GU301780.1/Thailand/2008

GU301781.1/Thailand/2009

72. FJ807899.1/Malaysia/2008

CP16:1-3 5-8/T hailand/2010

CP 16:4/T hailand $/ 2010$

CP $1: 4 / T$ hailand $/ 2010$

CP 7:6/T hailand $/ 2010$

CP13:2/T hailand/2010

CP1:1-3 5-8/T hailand/2010

CP7:1-5 7-8/T hailand/2010

CP9:1-8/T hailand/2010

CP10:1-8/T hailand/2010

12) CP11:1-8/T hailand/2010

CP13:1 3-8/T hailand/2010

GU301779.1/Thailand/2009

GQ905863.1/Thailand/2009

GU013529.2/S rilanka/2008

EF027136.1/India/2006

86. EU372006.1//ndia/2007

FJ807896.1/Taiwan/2009

FJ445511.2/Singapore/2008

FJ445510.2/Singapore/2008

EF027138.1/India/2006

FJ445428.2/S rilanka/2007

EF027137.1/India/2006

HQ456255.1/Kenya/2004-05

- HM045793.1/Central African Region/1986

- HM045822.1/Central African Republic/1978

100

- HM045823.1/Angola/1962

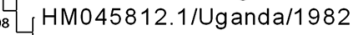

99 EF 027139.1/India/2000

HM0 45811.1/T anzania/1953

${ }_{100}$ HM045795.1/South Africa/1976

- AY424803.1/India/2003

HM045810.1/Thailand/1958

100 - $376613 /$ Tanzania/1953

97 HM045808.1/Thailand/1978

52 - HM045814.1/T hailand/1975

${ }_{9894}$ - HM045797.1/Indonesia/2010

[ HM0 45800.1/Philippines/1985

92 HM045789.1/T hailand/1988

${ }_{76}-\mathrm{HM} 045802.1 / \mathrm{T}$ hailand/1955

HM0 45807.1/Nigeria/1965

100 HM045820.1/Cote d Ivoire/1993

${ }_{60}$ AY726732.1/Senegal/1983

genotype

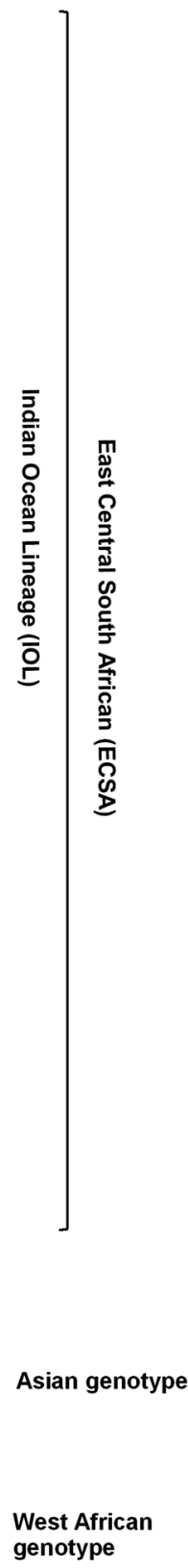

0.02

(b)

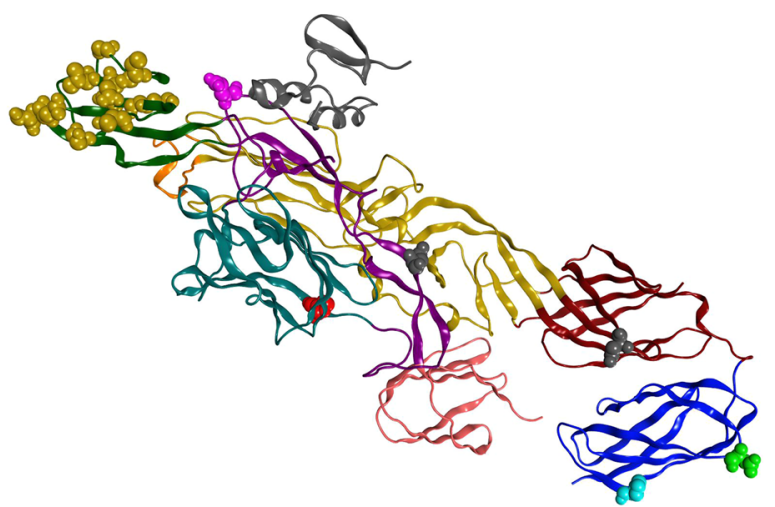


Table 1 Novel amino acid substitutions identified in the 56 chikungunya virus (CHIKV) clones derived from 7 patients

\begin{tabular}{|c|c|c|c|c|c|}
\hline Clinical isolates & Region & Polypeptide position $^{\mathrm{a}}$ & Protein position $^{\mathrm{b}}$ & 2008-2009 Thai outbreak strain & Amino acid substitution \\
\hline CP13-2 & $\mathrm{E} 2$ & 456 & 131 & $\mathrm{H}$ & $\mathrm{Y}$ \\
\hline CP16-4 & E2 & 572 & 247 & $\mathrm{E}$ & A \\
\hline CP7-6 & E1 & 1,113 & 304 & $\mathrm{P}$ & $\mathrm{L}$ \\
\hline CP16 (all clones) & E1 & 1,158 & 349 & $\mathrm{~N}$ & I \\
\hline
\end{tabular}

a, b Polypeptide and protein positions are in accordance with the published sequence of CHIKV complete genome (GenBank Accession no. AF369024.2)

plaque purification in Vero cells. Eight plaques were selected for each of the isolates: CP1:1-8, CP7:1-8, CP9:1-8, CP10:1-8, CP11:1-8, CP13:1-8, and CP16:1-8. The region containing the structural genes (capsid-E3E2-6k-E1) was amplified using primers published by Sreekumar et al. [13]. The amplicons were sequenced and aligned with the reference strain sequences available in GenBank (Fig. 1a). The E1-A226V and E2-I211T mutations, which provide a suitable background for CHIKV adaptation in Ae. albopictus [10, 11, 14, 15], were relatively well conserved among all clones obtained from the seven clinical isolates. Furthermore, several sequence variations were identified. We identified several unique amino acid substitutions that were not present in the strains responsible for the Thai outbreak in 2008-2009: E1-N349I in all CP16 clones; E1-P304L in clone CP7:6; E2-H131Y in clone CP13:2; and E2-E247A in clone CP16:4 (Table 1). These substitutions were conserved when compared to any strains reported in neighboring countries. Moreover, these are exposed on the surface of the molecule, suggesting that it may affect interaction of them with the other E monomers or antibodies (Fig. 1b) [16]. Phylogenetic analysis showed that all of the clinical isolates in 2010 formed a homogeneous cluster within a broad group, which also included the isolates from the Thai outbreak of 2008-2009 (ECSA genotype). Notably, all clones formed a branch that shared $>99 \%$ homology with the Indian Ocean Lineage (IOL), a relatively independent cluster within the ECSA genotype that caused an outbreak of unprecedented magnitude in 2005-2006 (Fig. 1a). This conclusion was also supported by the phylogenetic analysis based on $E 1$ gene sequence (data not shown). The CHIKV isolates from China (2010), India (2010-2011), and Cambodia (2011) also clustered within the IOL.

In conclusion, we isolated CHIKV derived from CHIKF-suspected patients in Ratchaburi Province of Thailand in 2010, and we report that CHIKV shows different characteristics when cultured in C6/36 cells, although it is not clear whether this is owing to the amino acid substitutions in the structural genes. Furthermore, CHIKV quasispecies are present in the Thai isolates from 2010, which include the clones harboring unique amino acid substitutions close to the immunodominant site
(Fig. 1b) [16]. However, as we cannot rule out the possibility of antigenic escape mutants, further analysis is required.

If we anticipate new outbreaks of chikungunya in Thailand, then we must increase CHIKV surveillance in disease-epidemic areas and deal with local outbreaks more effectively. Further studies of CHIKV isolated from either mosquitoes or chikungunya patients are needed.

Acknowledgments We would like to thank Naokazu Takeda, Surapee Anantapreecha and Yoshiharu Matsuura for their help with this study. This study was supported by the program of Japan Initiative for Global Research Network on Infectious Diseases by the Ministry of Education, Culture, Sports, Science, and Technology (MEXT) of Japan, a TropMed Grant and Dean fund year 2010 from the Faculty of Tropical Medicine, Mahidol University, and by a Grant-in-Aid for Young Scientists (B) (No. 25860407) from the MEXT of Japan.

Open Access This article is distributed under the terms of the Creative Commons Attribution License which permits any use, distribution, and reproduction in any medium, provided the original author(s) and the source are credited.

\section{References}

1. R. Pulmanausahakul, S. Roytrakul, P. Auewarakul, D.R. Smith, Chikungunya in Southeast Asia: understanding the emergence and finding solutions. Int. J. Infect. Dis. 15, e671 (2011)

2. J.E. Staples, R.F. Breiman, A.M. Powers, Chikungunya fever: an epidemiological review of a re-emerging infectious disease. Clin. Infect. Dis. 49, 942 (2009)

3. G. Rezza, L. Nicoletti, R. Angelini, R. Romi, A.C. Finarelli, M. Panning, P. Cordioli, C. Fortuna, S. Boros, F. Magurano, G. Silvi, P. Angelini, M. Dottori, M.G. Ciufolini, G.C. Majori, A. Cassone, CHIKV study group. Infection with chikungunya virus in Italy: an outbreak in a temperate region. Lancet. 370, 1840 (2007)

4. M. Dupont-Rouzeyrol, V. Caro, L. Guillaumot, M. Vazeille, E. D'Ortenzio, J.M. Thiberge, N. Baroux, A.C. Gourinat, M. Grandadam, A.B. Failloux, Chikungunya virus and the mosquito vector Aedes aegypti in New Caledonia (South Pacific Region). Vector. Borne. Zoonotic. Dis. 12, 1036 (2012)

5. Centers for disease control and prevention. Chikungunya in the caribbean (2014), http://wwwnc.cdc.gov/travel/notices/watch/chi kungunya-saint-martin. Accessed 07 May 2014

6. P. Rianthavorn, K. Prianantathavorn, N. Wuttirattanakowit, A. Theamboonlers, Y. Poovorawan, An outbreak of chikungunya in southern Thailand from 2008 to 2009 caused by African strains with A226V mutation. Int. J. Infect. Dis. 14S, e161 (2010) 
7. L. Dupuis-Maguiraga, M. Noret, S. Brun, R. Le Grand, G. Gras, P. Roques, Chikungunya disease: infection-associated markers from the acute to the chronic phase of arbovirus-induced arthralgia. PLoS. Negl. Trop. Dis. 6, e1446 (2012)

8. V.J. Lee, A. Chow, X. Zheng, L.R. Carrasco, A.R. Cook, D.C. Lye, L.C. Ng, Y.S. Leo, Simple clinical and laboratory predictors of Chikungunya versus dengue infections in adults. PLoS. Negl. Trop. Dis. 6, e1786 (2012)

9. P. Rianthavorn, K. Prianantathavorn, N. Wuttirattanakowit, A. Theamboonlers, Y. Poovorawan, An outbreak of chikungunya in southern Thailand from 2008 to 2009 caused by African strains with A226V mutation. Int. J. Infect. Dis. 14, e161 (2010)

10. M. Vazeille, S. Moutailler, D. Coudrier, C. Rousseaux, H. Khun, M. Huerre, J. Thiria, J.S. Dehecg, D. Fontenille, I. Schuffenecker, P. Despres, A.B. Failloux, Two Chikungunya isolates from the outbreak of La Reunion (Indian Ocean) exhibit different patterns of infection in the mosquito, Aedes albopictus. PLoS. One. 2, e1168 (2007)

11. K.A. Tsetsarkin, D.L. Vanlandingham, C.E. McGee, S. Higgs, A single mutation in chikungunya virus affects vector specificity and epidemic potential. PLoS. Pathog. 3, e201 (2007)

12. B. Pastorino, M. Bessaud, M. Grandadam, S. Murri, H.J. Tolou, C.N. Peyrefitte, Development of a TaqMan RT-PCR assay without RNA extraction step for the detection and quantification of African Chikungunya viruses. J. Virol. Methods 124, 65 (2005)

13. E. Sreekumar, A. Issac, S. Nair, R. Hariharan, M.B. Janki, D.S. Arathy, R. Regu, T. Mathew, M. Anoop, K.P. Niyas, M.R. Pillai, Genetic characterization of 2006-2008 isolates of Chikungunya virus from Kerala, South India, by whole genome sequence analysis. Virus. Genes. 40, 14 (2010)

14. K.A. Tsetsarkin, C.E. McGee, S.M. Volk, D.L. Vanlandingham, S.C. Weaver, S. Higgs, Epistatic roles of E2 glycoprotein mutations in adaption of chikungunya virus to Aedes albopictus and Ae. aegypti mosquitoes. PLoS. ONE. 4, e6835 (2009)

15. K.A. Tsetsarkin, R. Chen, M.B. Sherman, S.C. Weaver, Chikungunya virus: evolution and genetic determinants of emergence. Curr. Opin. Virol. 1, 310 (2011)

16. J.E. Voss, M.C. Vaney, S. Duquerroy, C. Vonrhein, C. GirardBlanc, E. Crublet, A. Thompson, G. Bricogne, F.A. Rey, Glycoprotein organization of Chikungunya virus particles revealed by X-ray crystallography. Nature. 468, 709 (2010) 